\title{
Optical characteristics of the OLED with microlens array film attachment
}

\author{
Hoang-Yan Lin*, Jiun-Haw Lee, ${ }^{1}$ Mao-Kuo Wei, ${ }^{2}$ Kuan-Yu Chen, ${ }^{1}$ \\ Sheng-Chih Hsu ${ }^{1}$, Yu-Hsuan $\mathrm{Ho}^{1}$, Chung-Yu Lin ${ }^{1}$ \\ ${ }^{1}$ Graduate Institute of Electro-Optical Engineering and Department of Electrical Engineering, \\ National Taiwan University, \\ No. 1, Sec. 4, Roosevelt Road, Taipei, 106 Taiwan, Republic of China \\ ${ }^{2}$ Department of Materials Science and Engineering, National Dong Hwa University, \\ No.1, Sec. 2, Da Hsueh Rd., Shoufeng, Hualien, 97401, Taiwan, Republic of China \\ *Phone: +886-2-3366-3663, Fax: +886-2-2367-7467, Email: hylin@cc.ee.ntu.edu.tw
}

\begin{abstract}
We investigated the luminance enhancement, spectral shift and image blur of the OLED with the microlens array film (MAF) attachment experimentally and theoretically. Higher density, larger curvature, and smaller diameter of the microlenses extracted more light from the substrate mode. The maximum improvements of the luminance at the normal direction and the total power were $42.5 \%(80 \%)$ and $45 \%$ (85\%) from our experimental (simulation) results, respectively. The differences between the theoretical and experimental results may come from the non-Lambertian radiation of OLED and the imperfection of the microlens array film. From observing the planar OLED, the peak wavelength is blue-shifted and the full width at the half maximum (FWHM) decreased with respect to increasing viewing angles due to the microcavity effect. When the MAF was attached, the spectral peak had a further blue shift (5 to $10 \mathrm{~nm}$ at different viewing angles) compared to that of the planar OLED and it came from the light extraction of the MAF from the substrate mode.

We also quantitatively investigated the "blur width" of the OLED with MAF attachment. Higher image blur was observed as accompanied with higher extraction efficiency which showed a tradeoff between the image quality and extraction efficiency. It means that the MAF attachment is more suitable for OLED lighting application, rather than display application. To reduce the image blur and keep the high extraction efficiency at the same time, we re-designed the arrangement of the microlens arrays on the film. In our optimized case, we found that the blur width can be reduced from $79 \mu \mathrm{m}$ to $9 \mu \mathrm{m}$, while the extraction efficiency is kept nearly the same. It shows a possibility to use the microlens array film on real OLED display for improving the extraction efficiency without image quality degradation.
\end{abstract}

\section{Objective and Background}

Organic Light-Emitting Devices (OLEDs) have attracted a lot of attention due to the advantages of wide viewing angle, self-emission, and low driving voltage [1]. However, the light extraction efficiency is limited to $20 \sim 30 \%$ due to the waveguiding effect of the glass substrate, organic materials, and ITO electrode. To improve the efficiency, the microlens array film (MAF) attached on the glass substrate has been used to outcouple more photons and this technique exhibits the advantages of easy fabrication and large-size capability [2]. However, the resultant spectral shift and the image blur effect may limit its application in OLED displays [3].

The objective of this paper is to quantitatively investigate the optical characteristics, which include external quantum efficiency (EQE), spectral shift, and image blur, for establishing a design rule for the MAF in OLED lighting and display applications, theoretically and experimentally. From our experimental results, a $45 \%$ EQE was achieved and a blue-shift in spectrum and image blur was observed. A 2-D numerical simulation program was established for obtaining simulation results which show similar trends with the experimental results.

The objective of this paper is to improve the light extraction efficiency of the OLED with human eye acceptable

Organic Light Emitting Materials and Devices XI, edited by Zakya H. Kafafi, Franky So, Proc. of SPIE Vol. 6655, 66551H, (2007) · 0277-786X/07/\$18 - doi: 10.1117/12.731521 
image blur. To quantitatively evaluate the degree of image blurring, we set up a criterion as the blur-width for the luminance distribution. The relationship between luminance improvement and image blur are discussed. Finally, a novel microstructure arrangement design is shown to gain sufficiently high efficiency enhancement and much less image blur than the traditional cases.

\section{Results}

In our OLED, we use $\mathrm{N}^{4}, \mathrm{~N}^{4}$-Di-naphthalen-2-yl- $\mathrm{N}^{4}, \mathrm{~N}^{4}$-di-naphthalen-1-yl-biphenyl-4,4'-diamine (TNB) as the hole-transport layer material and bis(10-hydroxyben-zo[h]quinolinato) beryllium (Bebq2) as the emitting and the electron-transport layer material. The device structure is: ITO $(110 \mathrm{~nm}) / \mathrm{TNB}(80 \mathrm{~nm}) / \mathrm{Bebq} 2(80 \mathrm{~nm}) / \mathrm{LiF}(0.7 \mathrm{~nm}) / \mathrm{Al}$ $(100 \mathrm{~nm})$. The glass substrate is $0.7 \mathrm{~mm}$. When the OLED device without any microlens array films was driven at a luminance of $714 \mathrm{~cd} / \mathrm{m}^{2}$, the peak wavelength and full width at half maximum (FWHM) of the spectrum were 516 and $95.3 \mathrm{~nm}$, respectively. The CIE coordinate of the device was $(0.2741,0.5324)$. In our simulations, we use the LightTools ${ }^{\circledR}$ which is software for designing an illuminant based on ray-tracing method.

Fig. 1 (a) and (b) show the experimental and simulation results of the luminance enhancement at the normal direction for the MAF with different coverage ratios (which is defined as the microlens area over the whole area) on our OLED, respectively. In Fig. 1 (a), each line represents different base shapes and arrangements of microlenses. We can observe that the enhancement ratio linearly increases with the coverage ratio. Besides, the smaller base area of the microlenses, the higher is the luminance enhancement. The best performance of $42.5 \%$ is SC35, $G=2.0$, which represents square base with gap $2 \mu \mathrm{m}$, because of its highest coverage ratio of $90 \%$ and smallest base area of $1250 \mu \mathrm{m}^{2}$. Fig. 1 (b) is the simulation results which show linear increase of enhancement ratio with increasing the coverage ratio and a similar trend with the experimental results. With increasing the density of the microlens array, the bumpy structure can destroy the planar waveguiding effect and the enhancement ratio is linearly correlated to the microlens area.

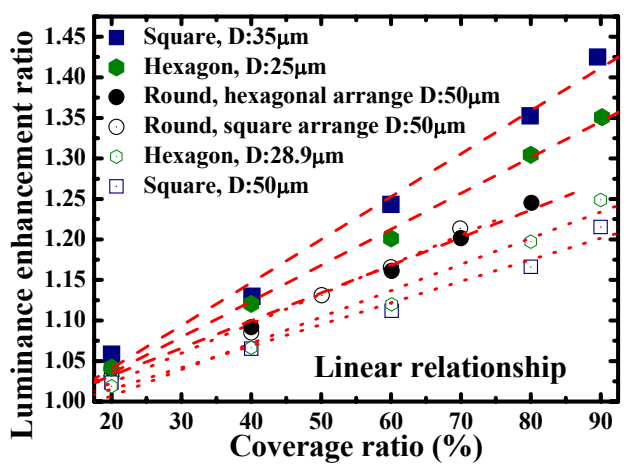

(a)

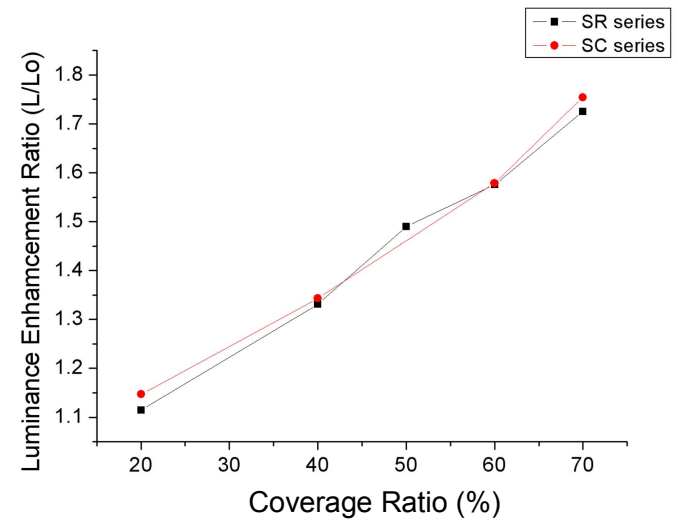

(b)

Fig. 1 (a) Experimental results, (b) simulation results of the OLED attached by MAF with different coverage ratios.

Fig. 2 (a), (b), (c), and (d) show the experimental results, simulation results, and 1-D simulation ray tracings for the MAF with different microlens height on our OLED. In Fig. 2(a), each line represents different base shapes and arrangements of microlenses. We can observe that the enhancement ratio increases rapidly then achieves a saturation value when the PR height increases. Fig 2(b) also shows a similar trend. Fig. 2(c) and (d) shows the simulation result of the ray tracing with different heights: (c) the same as and (d) $40 \%$ of the radius. The illumination source is placed $100 \mathrm{~nm}$ higher than the reflective surface. Total 200 traced rays are spread in the forward $120^{\circ}$ cone to show qualitative optical behaviors. We can clearly see that ray number coupled out in the Fig. 2(c) is larger than that in Fig 2(d) since the curvature of the microlens is larger when the microlens is higher. 

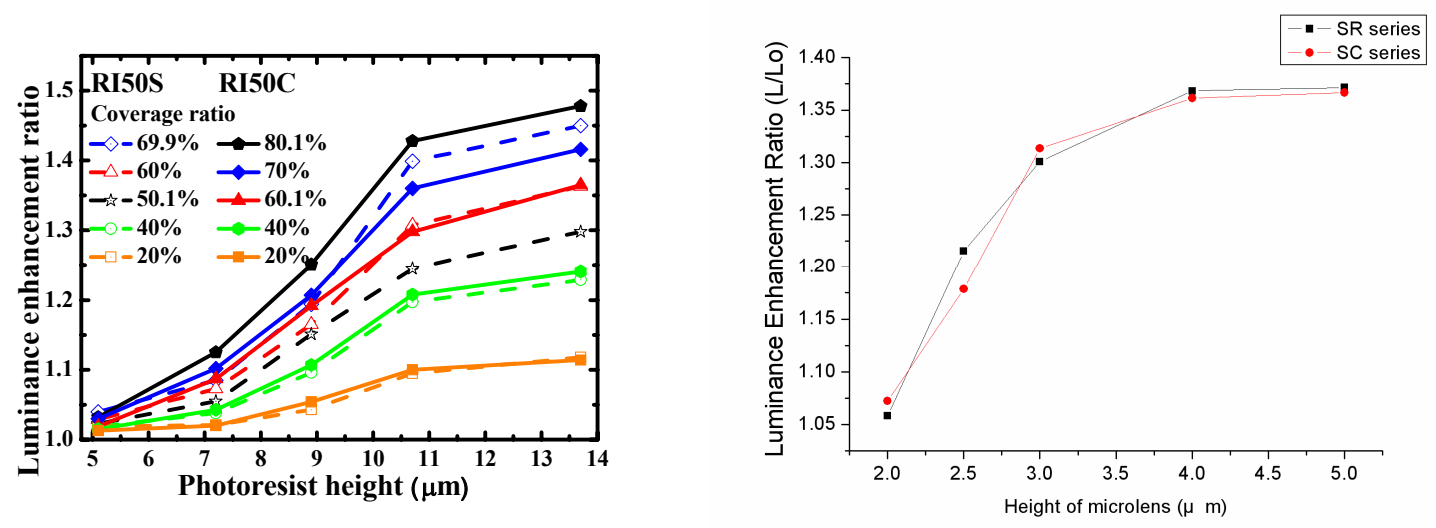

(a)

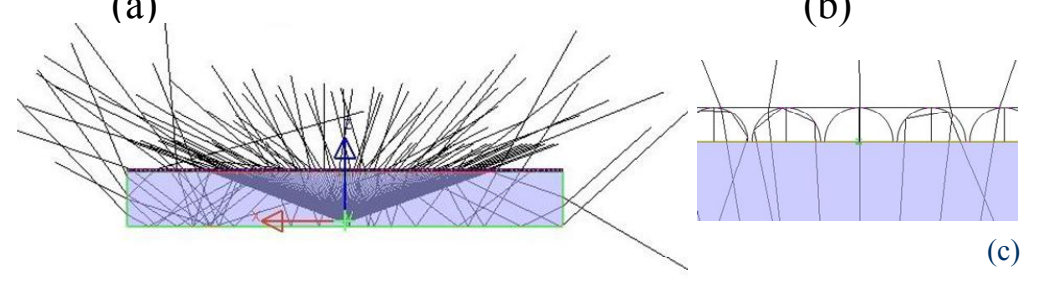

(c)

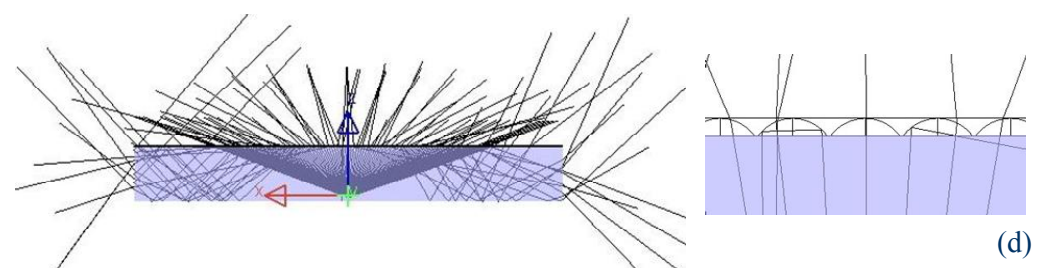

Fig. 2 (a) Experimental results, (b) simulation results of the OLED attached by MAF with different microlens heights. (c) and (d) show the ray-tracing simulation results with different microlens heights.

Fig. 3 shows the spectral at different viewangles for the planar- and the MAF-OLED. For our planar-OLED, a spectral peak blue shift from $516 \mathrm{~nm}$ in normal direction to $508 \mathrm{~nm}$ in $80^{\circ}$ was observed, which is typical phenomenon in some OLED and can be explained by Fabry-Perot equation [4]. This also implies the waveguiding mode exhibits shorter wavelength than the outcoupling mode. Fig. 3 (b) shows the MAF-OLED which shows a blueshift from 512 to $501 \mathrm{~nm}$ with the viewangles from $0^{\circ}$ to $80^{\circ}$. That means the MAF can effectively outcoupled the waveguiding light. These results also reflect that the spectral shift of the MAF-OLED may be an issue when using for the display application.
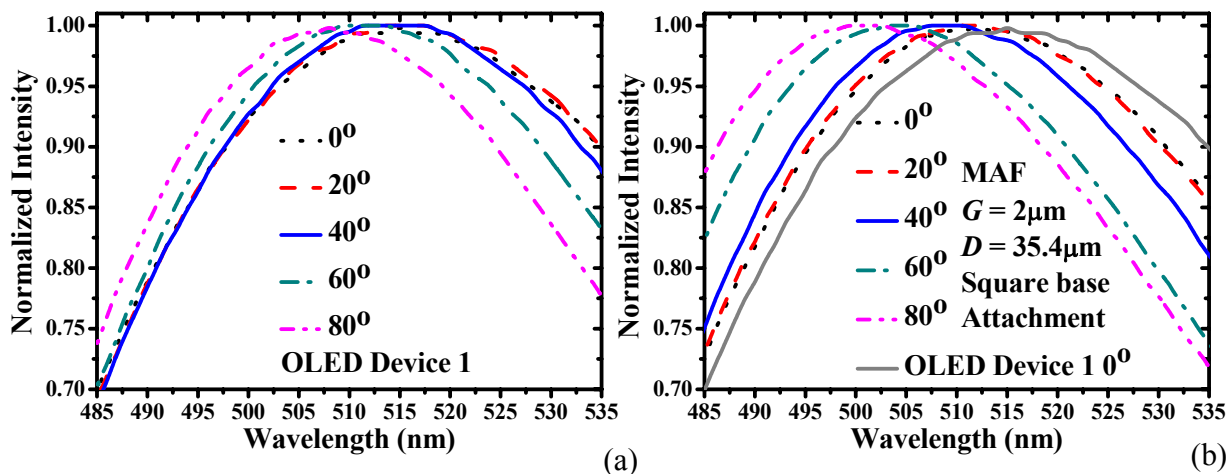

Fig. 3 Spectra at different viewangles of (a) planar- and (b) MAF-OLED. 
Fig. 4 shows the experimental results ((a) and (b)) and simulation results ((c) and (d)) of the 2-D image with the planar- ((a) and (c)) and MAF-OLED ((b) and (d)). Fig. 4 (a) and (b) are taken under the optical microscope with a small pixel OLED emission $\left(188 \times 188 \mu \mathrm{m}^{2}\right)$. We can clearly see that the intensity profile of the MAF-OLED is modulated by the microlens structure which results in serious blur effect and degrades the image quality in display applications.

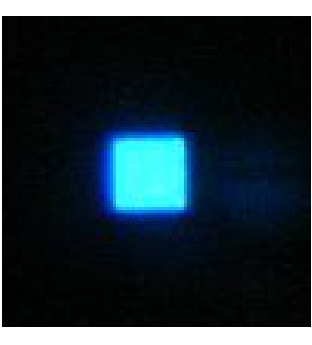

(a)

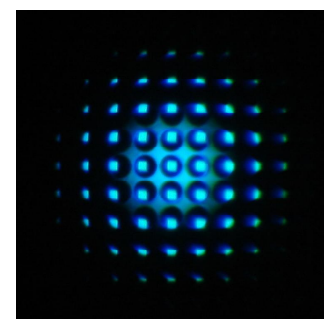

(b)

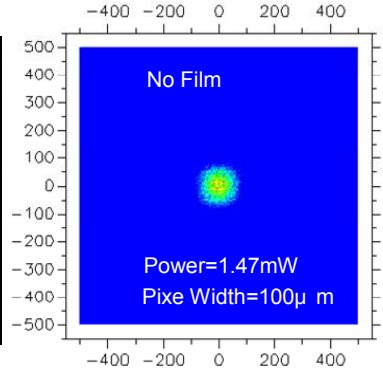

(c)
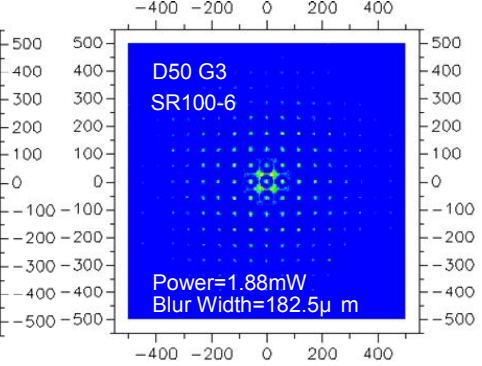

(d)

Fig. 4 2-D image of the experimental and simulation results for the planar- and MAF-OLED.

The traditional approach is to uniformly distribute the microstructures on the substrate. From the above observation, we propose an innovative microstructure arrangement design, which we called the "defect" microstructure array: the microstructures upon the pixels are removed to increase the forward parallel ray flux. These defects prevent the inclined rays to be extracted from the neighboring pixels, so they reduce the extraction efficiency but also the crosstalk or blur. The remaining microstructures keep the extraction ability of the inclined rays. To sum up, light extraction efficiency is kept the quite same but the image blur can be reduced as compared with the traditional approach.

To demonstrate the influence of the "defect" microstructure arrangement, we establish a series of optical model. Figure 5 shows a group of RGB sub-pixels of the AMOLED without microstructure and the corresponding luminance distribution along $\mathrm{x}$ - and $\mathrm{y}$-axis.

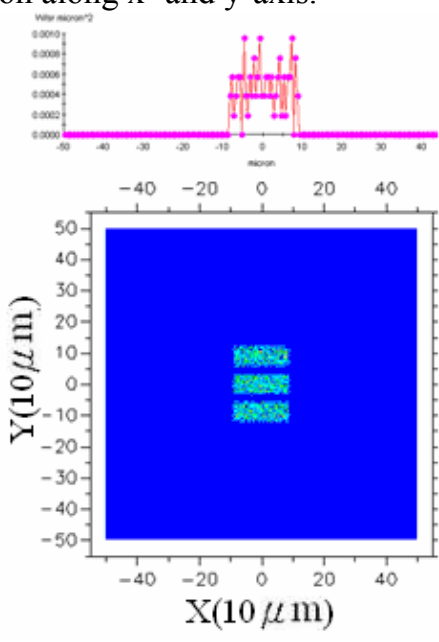

(a)

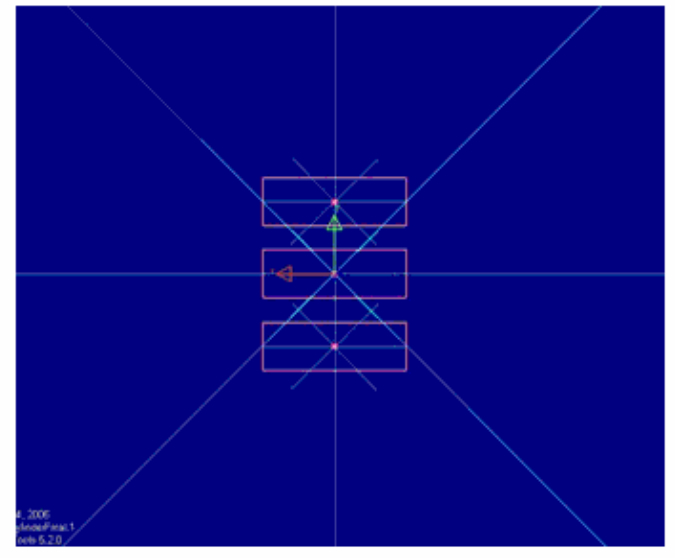

(b)

Figure 5 (a) the simulated image of the three "ON" OLED sub-pixels on the observation plane and (b) the configuration of the three "ON" OLED sub-pixels without microlens array film attachment.

In Figure 6, with the traditional microstructure array applied, the luminance distribution of $\mathrm{x}$ - and $\mathrm{y}$-axis are spread outside the pixel. That is, the three sub-pixel image is suffered from image blur. 


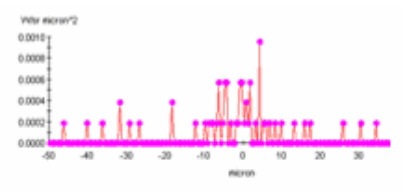

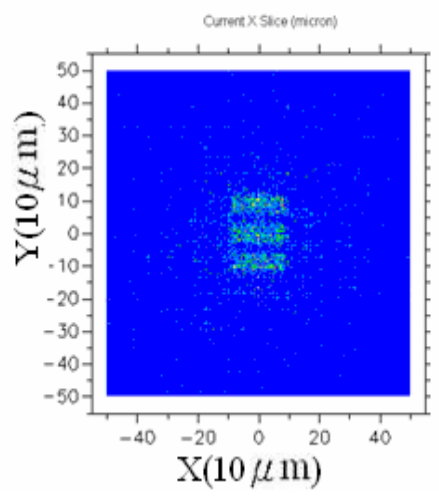

(a)

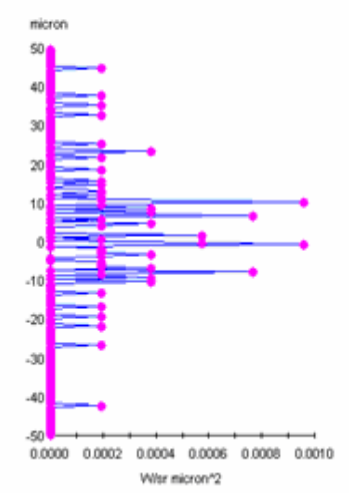

What eictorn?

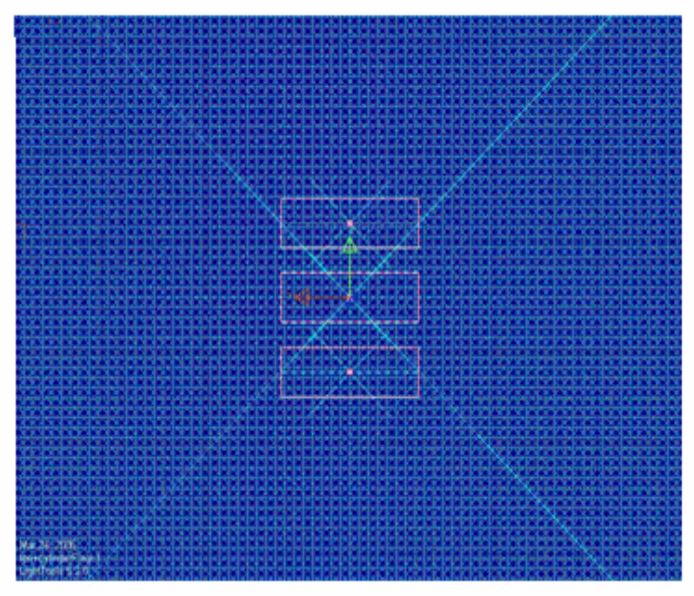

(b)

Figure 6 (a) the simulated image of the three "ON" OLED sub-pixels on the observation plane and (b) the configuration of the three "ON" OLED sub-pixels $(157 \mu \mathrm{m}$ x $39 \mu \mathrm{m})$ with full microlens $(\phi=10 \mu \mathrm{m}$, gap=2 $\mu \mathrm{m})$ array film attachment.

In Figure 7, the novel "defect" microstructure arrangement is shown to be with little image blur.

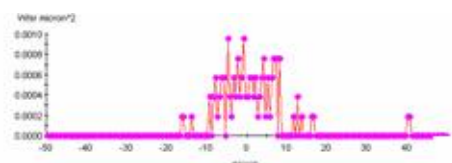

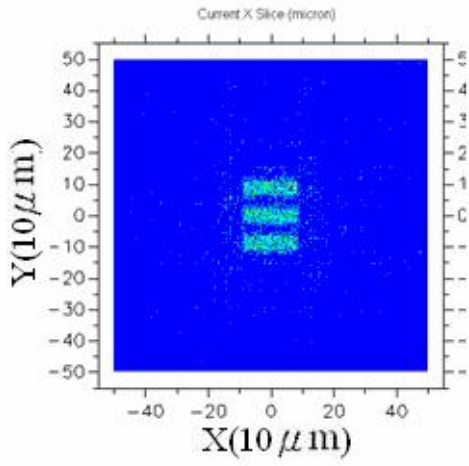

(a)

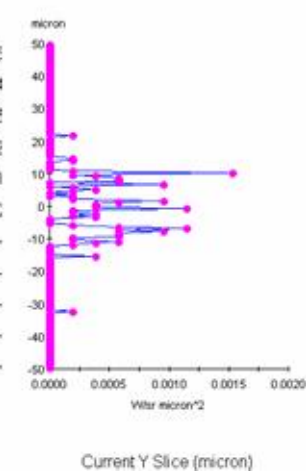

Current Y Slice (micron)

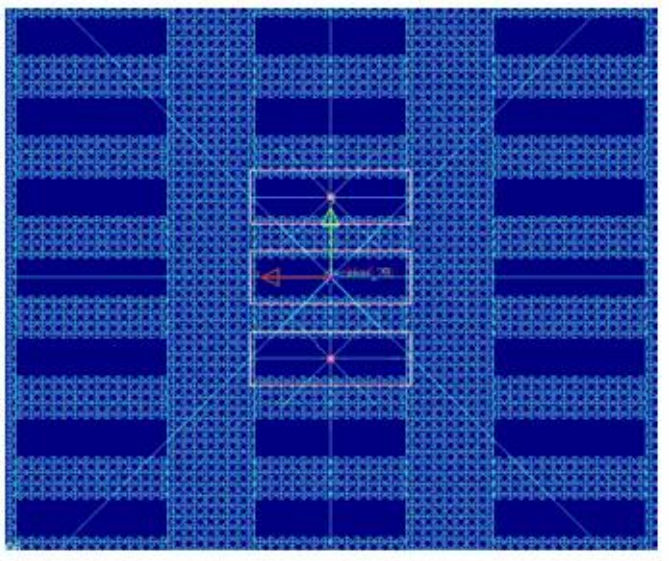

(b)

Figure 7 (a) the simulated image of the three "ON" OLED sub-pixels on the observation plane and (b) the configuration of the three "ON" OLED sub-pixels $(157 \mu \mathrm{m} \times 39 \mu \mathrm{m})$ with defect microlens $(\phi=10 \mu \mathrm{m}$, gap=2 $\mu \mathrm{m})$ array film attachment.

Figure 8 shows the relative radiant intensity vs. view angle. The normal-direction (zero degree) luminance enhancement of the novel "defect" microstructure arrangement is slightly larger than that of the traditional microstructures. However, the power efficiency is summed up to be a little bit less than that of the traditional case full of microstructure. 


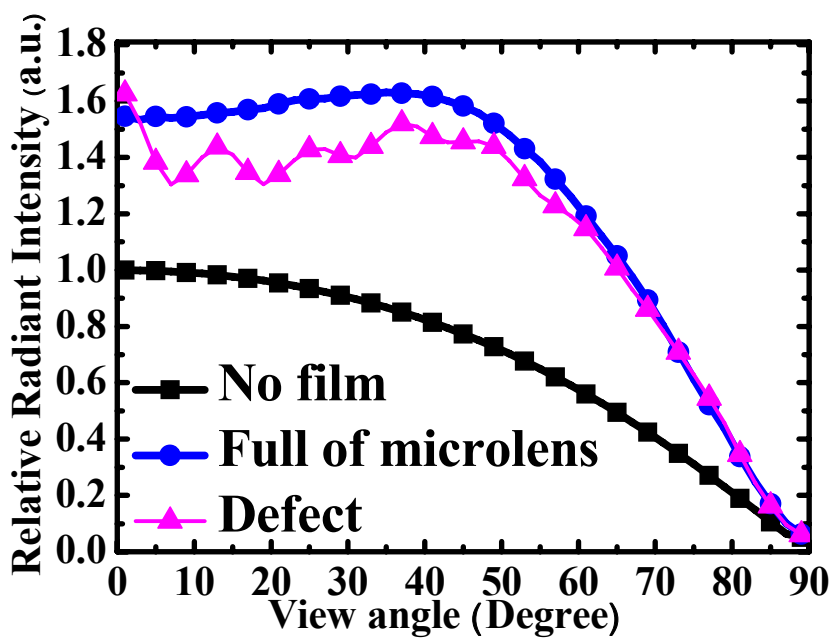

Figure 8 Relative radiant intensity vs. view angle

In Table 1, we summarize the relationship of relative power, luminance, $\mathrm{x}$ - and $\mathrm{y}$-axis blur-width for the cases without, with traditional and with "defect" $157 \mu \mathrm{m}$ x $39 \mu \mathrm{m}$ microstructure array films. The case with "defect" microstructure array exhibits higher relative luminance than that of the traditional case, keep certain high relative power level, and cuts down blur-widths from $124 \mu \mathrm{m}$ and $79 \mu \mathrm{m}$ to $24 \mu \mathrm{m}$ and $9 \mu \mathrm{m}$ (to the human-eye negligible range) in both the $\mathrm{x}$ - and y-axis, respectively.

Table 1 The relationship between relative power, luminance, $x$ - and y-axis blur-width

\begin{tabular}{|c|c|c|c|c|}
\hline Original pixel=157 x 39 & \multirow{2}{*}{ Relative power } & \multirow{2}{*}{ Relative luminance } & Blur-width (X) & Blur-width (Y) \\
\cline { 4 - 5 } Unit (micron) & & & Unit (micron) & Unit (micron) \\
\hline w/o microlens $\boldsymbol{C R}=0 \%$ & 1 & 1 & x & x \\
\hline w/ microlens $\boldsymbol{C} \boldsymbol{R}=54.5 \%$ & 1.93 & 1.55 & 124 & 79 \\
\hline defect array $\boldsymbol{C R}=37.8 \%$ & 1.79 & 1.63 & 24 & 9 \\
\hline
\end{tabular}

\section{Impact}

Planar light emitting displays are usually suffered from low light extraction efficiency due to total internal reflection and surface texturing is the common approach to circumvent it. We quantitatively investigate the optical characteristics of the MAF-OLED. Although the EQE can be effectively increased, it results in spectral shift and the blur effect which is crucial for display application. On the other hand, this MAF-OLED is promising for lighting application. However, surface texturing deteriorates the image quality. This work emphasizes the importance of microstructure arrangement with respect to the pixel layout. The dilemma between luminance improvement and induced image-blur effect can be solved by using this novel design.

\section{Acknowledgments}

The authors gratefully acknowledge the financial support given by the National Science Council of the Republic of China under the project No. NSC 95-2221-E-259-030, NSC 96-2221-E-002-126 and NSC 95-2221-E-002-271-.

\section{References}

1. C. W. Tang et. al., Appl. Phys. Lett., 51, 913 (1987).

2. M.-K. Wei et. al., Opt. Express, 12, pp. 5777 (2004).

3. H. Peng et. al., J. Display Technology, 1, 278 (2005).

4. J. H. Lee et. al., J. Display Technology, 2, 130 (2006). 